\title{
Diffraction-Enhanced Imaging of Musculoskeletal Tissues Using a Conventional X-Ray Tube
}

\author{
Carol Muehleman, Ph.D. ${ }^{a}$, Jun Li, M.D. ${ }^{a}$, Dean Connor, Ph.D. ${ }^{b}$, Christopher Parham, M.D.C, \\ Etta Pisano, M.D.C, and Zhong Zhong, Ph.D. ${ }^{\mathrm{b}}$ \\ aRush University Medical Center, Department of Biochemistry, Chicago, IL. \\ bNational Synchrotron Light Source, Brookhaven National Laboratory, Upton, N.Y. \\ cBiomedical Research Imaging Center and Lineberger Comprehensive Cancer Center, Chapel Hill, \\ NC
}

\begin{abstract}
Rationale and Objectives-In conventional projection radiography, cartilage and other soft tissues do not produce enough radiographic contrast to be distinguishable from each other. Diffraction-enhanced imaging (DEI) uses a monochromatic x-ray beam and a silicon crystal analyzer to produce images in which attenuation contrast is greatly enhanced and $\mathrm{x}$-ray refraction at tissue boundaries can be detected. Here we test the efficacy of conventional x-ray tube-based DEI for the detection of soft tissues in experimental samples.
\end{abstract}

Materials and Methods-Cadaveric human tali (normal and degenerated), knee, and thumb were imaged by DEI by using a conventional x-ray tube and DEI set-up which includes a double silicon crystal monochromator and a silicon crystal analyzer positioned between the imaged object and a detector.

Results-DEI images of the cadaveric tali allowed visualization of cartilage and its specific level of degeneration for each specimen. There was a significant correlation between grade of cartilage integrity as assessed through tube-DEI images in comparison to their respective histological sections $(r=0.97 ; p=0.01)$. Images of the intact knee showed the articular cartilage edge of the femoral condyle even though superimposed by the tibia. In the thumb image, it was possible to visualize articular cartilage, tendon, and other soft tissues.

Conclusion-DEI, based on a conventional x-ray tube, allows the visualization of skeletal and soft tissues simultaneously. Although more in-depth testing and optimization of the DEI set-up must be carried out, these data demonstrate a proof of principle for further development of the technology for future clinical imaging.

\section{Keywords}

diffraction-enhanced imaging; phase contrast imaging; cartilage imaging; DEI; osteoarthritis; soft tissue imaging

(C) 2009 The Association of University Radiologists. Published by Elsevier Inc. All rights reserved.

Corresponding author: Carol Muehleman, Ph.D., Department of Biochemistry, Rush University Medical Center, Cohn Room 541,1735 W., Harrison St. 60612, Email: carol_muehleman@rush.edu, Phone: 312-942-6780, FAX: 312-942-3053.

Publisher's Disclaimer: This is a PDF file of an unedited manuscript that has been accepted for publication. As a service to our customers we are providing this early version of the manuscript. The manuscript will undergo copyediting, typesetting, and review of the resulting proof before it is published in its final citable form. Please note that during the production process errors may be discovered which could affect the content, and all legal disclaimers that apply to the journal pertain. 


\section{INTRODUCTION}

Phase contrast imaging techniques are based on the utilization of information arising from the modification of amplitude and phase of $\mathrm{x}$-rays as they traverse an object. This allows for the detection of subject contrast due to tissue properties such as refraction. This contrast does not depend upon x-ray attenuation, as is the case with contrast in conventional radiography. Thus, tissue contrast that is difficult to detect through $\mathrm{x}$-ray attenuation, particularly at high energies where radiation dose is comparatively reduced, may be detected with phase contrast techniques. This includes soft tissues which do not have the composition to provide the necessary attenuation of $\mathrm{x}$-rays and require techniques which exploit $\mathrm{x}$-ray refraction at tissue boundaries in order to be visualized. One such technique is based on a system in which an analyzer crystal is positioned between the object and the detector. This allows only those $\mathrm{x}$-rays satisfying the Bragg condition to be diffracted to the detector. Changes in $\mathrm{x}$-ray reflection angle are converted to changes in X-ray intensity, through an intensity vs. reflection angle (measured in microradians) curve, described as the "rocking curve". By altering the angle of the analyzer, it is possible to record different refraction angles and thus extract both refraction and absorption characteristics. $1,2,3,4,5,6,7,8$

What renders this technology pertinent to the study of joint disease is that soft tissues, including articular cartilage, meniscus, tendon, and ligament are detected with reasonable clarity and contrast. Furthermore, it has been used to detect pathological changes within these tissues, even at early stages of disease. The drawback has been that DEI has, up until now, been used exclusively with synchrotron sources of $\mathrm{x}$-rays, rendering it impractical for routine clinical use. This led to the development of a system which could use DEI technology, but with a compact $\mathrm{x}$-ray source such as a commercially available tungsten tube (tube-DEI).

A practical application of DEI is in the detection of musculoskeletal lesions and cartilage lesions characteristic of osteoarthritis (OA). Because conventional radiography renders articular cartilage virtually invisible and because magnetic resonance imaging (MRI) still has some drawbacks i.e. insufficient resolution for early lesions and low sensitivity for chondrocalcinosis ${ }^{9}$, there is room for complementary technology such as DEI which exploits different tissue characteristics. Here we demonstrate the efficacy of an experimental model of tube-DEI for the visualization of cartilage and cartilage lesions in human tali and in intact cadaveric human joints.

\section{MATERIALS AND METHODS}

\section{Specimens}

The specimens imaged consisted of 12 human cadaveric tali ( 3 normal and 3 at each grade of cartilage degeneration, as described below) and a cadaveric human knee and thumb. The tali and knee were obtained from the Gift of Hope Organ and Tissue Donor Network of Illinois (with Institutional Review Board Approval). The thumb was obtained from the dissection laboratory of the first year medical students and it was returned to the cadaver after use. These specimens had been formalin-preserved prior to imaging, as we have previously shown that formalin fixation does not affect DEI imaging ${ }^{8}$. Tali were graded according to a macroscopic visual scale ${ }^{10}$ as follows: grade $0=$ normal, undisturbed articular cartilage surface; grade $1=$ fibrillated cartilage surface; grade 2 = fissured or ulcerated cartilage; grade 3 = cartilage eroded down to subchondral bone. Three tali for each grade were chosen at random, out of a larger sample of 100 tali, for the study. 


\section{Imaging Set-Up}

The x-ray source for the DEI system (Fig 1) is a Comet MXR-160HP/20 x-ray tube (Comet AG, Flamatt, Switzerland), with a stationary tungsten anode and a focal spot size of $0.4 \mathrm{~mm}$. A Titan 160 x-ray system (GE Inspection Technologies, Ahrensburg, Germany), with a maximum voltage of $160 \mathrm{kV}$ and $1 \mathrm{~kW}$ total power, powered the anode. A $2.0 \mathrm{~mm}$ thick tantalum collimator with a $25 \mathrm{~mm}$ wide by $1 \mathrm{~mm}$ high aperture was placed over the exit window of the $\mathrm{x}$-ray tube to create a fan beam ${ }^{12}$.

A monochromator was built using a single perfect float zone silicon crystal (Shaw Monochromators, Riverton, KS) of the 333 reflection type measuring $70 \mathrm{~mm}$ by $35 \mathrm{~mm}$ by 10 $\mathrm{mm}$. The monochromator was placed $100 \mathrm{~mm}$ from the $\mathrm{x}$-ray tube. The incident angle of the fan beam on the monochromator crystal was 5.7 degrees to select the $\mathrm{K} \alpha 1(59.318 \mathrm{keV})$ characteristic emission line of tungsten. Because of the source divergence and the monochromator's crystal size, the monochromator also reflected the K $\alpha 2(57.982 \mathrm{keV})$ emission line ${ }^{12}$.

\section{Imaging}

Each sample, at a distance of $650 \mathrm{~mm}$ from the $\mathrm{x}$-ray tube, was moved through the $\mathrm{x}$-ray beam using a translation stage (Newport Corporation, Irvine, CA). A silicon analyzer crystal measuring $150 \mathrm{~mm}$ by $60 \mathrm{~mm}$ by $10 \mathrm{~mm}$ was placed behind the sample and tuned to an angle of 5.7 degrees with respect to the imaging beam. The analyzer is the same type as used for synchrotron DEI studies at the National Synchrotron Light Source (Upton, NY, USA) ${ }^{1}$.

All images were acquired using a Fuji ST-VI general purpose imaging plate (Fuji Medical Systems, Stamford, CT) that was placed perpendicular to the post-analyzer crystal x-ray beam. The imaging plates were digitized using a Fuji BAS-2500 imaging plate reader (Fuji Medical Systems, Stamford, CT) at a resolution of 50 micrometers. This detector plate was selected because of its fixed noise for long exposure times and its detection efficiency at $59 \mathrm{keV}$. The imaging plate was scanned using a translation stage (Newport Corporation, Irvine, CA) in the opposite direction of the sample stage to form a radiograph of the sample using the fan beam. The detector and sample scanning methods have been previously described ${ }^{12}$. A surface dose of $0.07 \mathrm{mGy}$ was used for the DEI image.

\section{Histology}

Macroscopic grades were verified through histological sectioning of representative regions on the tali. Because serial sectioning of entire tali is neither reasonable nor necessary, representative regions were processed for microscopic examination by dehydration in a series of increasing alcohol concentrations, followed by paraffin infiltration and embedding. Several 5 - $\mu \mathrm{m}$ serial sections were made and adjacent sections were stained with Safranin O/Fast green $^{11}$ and examined under magnifications of 8, 10, 40, and 100X.

\section{Image grading and statistics}

Images were converted to jpg files and displayed on a Dell UltraSharp monitor. Two readers (CM, an anatomist and experienced DEI image reader, and JL, an orthopedic surgeon and experienced DEI image reader) graded the DEI images blindly according to the same modified Collin's scale ${ }^{10}$ used for the gross morphological and histological grading of the specimens. Correlation between tube-DEI grades and gross morphological and histological grades were made using Spearman's test. Statistical significance was taken at $\mathrm{p} \leq 0.05$. 


\section{RESULTS}

\section{Tali}

Representative results of tube-DEI for human tali are shown in Fig. 2. Spearman's correlation test showed very high correlation between grade of cartilage integrity as assessed through tubeDEI images in comparison to the respective histological sections, with $r=0.97(p=0.01)$. The only tube-DEI score that did not exactly match the gross and histological grade was one talus displaying grade 1 degeneration. For this specimen, there were two focal regions of superficial fibrillation which were not detected in the respective tube-DEI image by the readers. This is explained further below.

For normal cartilage, displaying no signs of degeneration (Fig. 2a), the DEI images show a homogeneous signal throughout the articular cartilage - no imperfections are visible. The Safranin O histological sections (below their respective tube-DEI images) show corresponding normal cartilage with intact articular surfaces. Fig. $2 b$ shows examples of grade 1 cartilage. The slight fibrillations noted in the histological sections are not well depicted in their respective tube-DEI images. Although the articular borders are not as crisply demarcated as those in the tube-DEI images of the grade 0 specimens of Fig. 1, this, and subtle heterogeneities within the cartilage, are the only subtle differences. It must be kept in mind that the tube-DEI image represents the entire thickness of the talus from anterior to posterior whereas the histological section is a $5-\mu \mathrm{m}$ slice through a focal fibrillated region magnified $\mathrm{x} 10$.

Fig. 2c demonstrates how the isolated fissure (grade 2) seen in the histological section is identifiable in its DEI image. In the tube-DEI image, two contrast heterogeneities seen to the right of the fissure are represented by the irregularities in the histological section. Again, because the tube-DEI image is taken through the entire depth of the talus, from anterior to posterior, and because the talus has an innate curvature, irregularities seen on the histological surface may actually be seen deeper into the cartilage in the corresponding tube-DEI image. Focal erosion of cartilage (grade 3) is identifiable in the tube-DEI images of Fig. 2d. In the upper portion of the figure, it can be seen that if there is absence of cartilage in a region lying in the same direction as the x-ray beam. This complete cartilage loss is visualized as such in its tube-DEI image by loss of x-ray contrast. However, if there is only focal cartilage loss in the path of the x-ray beam, the DEI image will be representative of the cartilage status throughout the path of the beam as seen in the lower image of Fig. 2d. Here we see the cartilage lesions as contrast heterogeneities.

\section{Knee and Finger}

Fig. 3 shows a tube-DEI image of an intact human cadaveric knee joint in which the edge of the articular cartilage is clearly visible on the condyle on the left and in part on the condyle on the right. Of particular note is that the articular edge of the cartilage is identifiable even when the cartilage is superimposed by bone due to the positioning of the joint in relation to the $\mathrm{x}$ ray beam.

Fig. 4 shows a tube-DEI image of an intact cadaveric human thumb in which the flexor tendon, extensor tendon, articular cartilage, fat pad with collagenous network, and nail are visible.

\section{DISCUSSION}

This is the first report on the efficacy of a conventional x-ray tube- DEI system for the imaging of articular cartilage and musculoskeletal tissue. In this report we have demonstrated that tubeDEI allows visualization of the articular cartilage, simultaneous with the bone, of disarticulated and intact synovial joint surfaces. Tube-DEI operates in the same manner as the synchrotronbased DEI system in that, through use of a monochromator and an analyzer crystal, $\mathrm{x}$-ray 
refraction information, in addition to the x-ray attenuation of conventional radiography, is available. It is evident that, by taking an image at the half-intensity points of the rocking curve, where x-ray refraction is greatest, soft tissue boundaries are readily identifiable. This is of particular relevance in the diagnosis of articular cartilage lesions which generally begin at the articular border, thus compromising this tissue boundary.

We have applied tube DEI technology to the problem of cartilage imaging for two major reasons. First, conventional radiography is the gold standard for the diagnosis of arthritis by providing information on pathological bone changes and joint space narrowing (which is a reflection of cartilage loss, as soft tissues are virtually invisible with conventional radiography). However, by the time enough cartilage has been eroded from the articular surface to result in narrowing of the joint space, the damage is both severe and beyond any possible processes of repair. This includes both innate repair (which may be nearly negligible) and changes in lifestyle/habits that contribute to cartilage damage. Second, although MRI is quite valuable in depicting cartilage and moderate to large cartilage lesions, it is still open to interpretation ${ }^{13}$, and early cartilage changes such as fibrillation and superficial damage are not detectable through this technology. Thus there is still need for other technologies, particularly those which allow detection of pathologies that provides more detailed data, perhaps at a time early enough to intervene and change disease course. DEI is one such technology.

The images of human tali, both with and without cartilage degeneration, demonstrate the ability of DEI to detect tissue boundaries. Any interruption in the articular border was reflected as such and to the exact extent of this interruption as verified histologically. This is the first step in verification of tube-DEI as a possible tool to identify early cartilage lesions, although the earliest of these lesions, i.e. superficial fibrillation, was not always detected. There are a couple of reasons for this. First, we have used a planar imaging set-up which produces an image through the entire thickness of the specimen. This inevitably results in the superimposition of small cartilage defects by regions of normal cartilage, thus masking these tiny imperfections. Although computed tomography would solve this problem, tube-based-CT-DEI technology is not yet developed. However, most fibrillations (grade 1 lesions) and all fissures (grade 2 lesions) and more severe lesions were visible with tube-DEI. These results are similar to those we previously reported for synchrotron-based DEI of human tali ${ }^{14,15}$ thus demonstrating that the technology can be transferred to a non-synchrotron-based system.

The identification of cartilage borders within the intact cadaveric human knee is remarkable in that the cartilage was superimposed by bone tissue of the tibia. This is a perfect illustration of the ability of DEI to detect x-ray refraction at borders between tissues, particularly those of different refractive indices, thus rendering the technology well-suited for resolving soft and hard tissues, a feat not possible with conventional radiography.

Identification of the articular cartilage, tendons, muscle, and collagenous network of the human thumb sample surely illustrates the profound clinical application of tube-DEI technology. It remains to be seen if tube-DEI can ever be used as the gold standard for musculoskeletal imaging as conventional attenuation radiography is currently used. However, the images presented here should stimulate further development and investigation into tube-DEI technology. This, of course, must include reduction in imaging times to be clinically viable ${ }^{12}$.

The results presented here are similar to those reported earlier by us using synchrotron $\mathrm{x}$ rays $8,14,15$, thus demonstrating that tube-DEI is not innately dependent upon an extremely high flux source of x-rays. Also, because the results have been obtained with suboptimal equipment, i.e. equipment which can be greatly improved upon in the clinical setting such as a more 
efficient detector and an x-ray tube of greater energy (thus even further decreasing radiation dose), we are optimistic that issues such as long imaging times can be vastly improved upon.

\section{Acknowledgement}

This work was supported by NIH grant R01 AR48292-05.

\section{REFERENCES}

1. Chapman D, Thomlinson W, Johnston RE, et al. Diffraction enhanced x-ray imaging. Phys Med Biol 1997;42:2015-2025. [PubMed: 9394394]

2. Muehleman C, Chapman LD, Kuettner KE, et al. Radiography of rabbit articular cartilage with diffraction enhanced imaging. Anat Rec 2003;272A:392-397.

3. Muehleman C, Majumdar S, Arfelli F, et al. Radiographic detection of structural orientation in articular cartilage. Osteoarthritis Cartilage 2004;12:97-105. [PubMed: 14723869]

4. Muehleman C, Li J, Wernick M, et al. Yes you can see cartilage with x-rays: diffraction enhanced imaging of cartilage and bone. J Musculoskelet Neuronal Interact 2004;4:369-370. [PubMed: 15758263]

5. Muehleman C, Li J, Zhong Z, et al. Multiple-image radiography human soft tissue. J Anat 2006;208:115-124. [PubMed: 16420384]

6. Muehleman C, Li J, Zhong Z. Preliminary study on diffraction enhanced imaging for a canine model of cartilage damage. Osteoarthritis Cartilage 2006;14:882-888. [PubMed: 16621619]

7. Lewis RA. Medical phase contrast x-ray imaging: current status and future prospects. Phys Med Biol 2004;49:3573-3583. [PubMed: 15446788]

8. Mollenhauer JA, Aurich ME, Zhong A, et al. Diffraction enhanced x-ray imaging of articular cartilage. Osteoarthritis Cartilage 2002;10:163-171. [PubMed: 11869076]

9. Abreu M, Johnson K, Chung CB, et al. Calcification in calcium pyrophosphate dihydrate (CPPD) crystalline deposits in the knee: anatomic, radiographic, MRI, and histologic study in cadavers. Skeletal Radiol 2004:3392-3988.

10. Muehleman C, Bareither D, Huch, et al. Prevalence of degenerative morphological changes in the joints of the lower extremity. Osteoarthritis Cartilage 1997;5:1-15. [PubMed: 9010874]

11. Rosenberg L. Chemical basis for the histological use of Safranin O in the study of articular cartilage. J Bone Joint Surg Am 1971;53:69-82. [PubMed: 4250366]

12. Parham CA, Zhong A, Connor D, Chapman D, Pisano ED. Design and implementation of a compact low-dose diffraction enhanced medical imaging system. Academic Radiology. in press

13. Burstein D, Gray ML. Is MRI fulfilling its promise for molecular imaging of cartilage in arthritis? Osteoarthritis Cartilage 2006;14:1087-1090. [PubMed: 16901724]

14. Li J, Williams JM, Zhong Z, et al. Reliablity of Diffraction Enhanced Imaging for Assessment of Cartilage Lesions, Ex Vivo. Osteoarthritis and Cartilage 2005;13:187-197. [PubMed: 15727884]

15. Li J, Zhong Z, Lidtke R, et al. Radiography of Soft Tissue of the Foot and Ankle with Diffraction Enhanced Imaging. J Anat 2003;202:463-470. [PubMed: 12739623] 


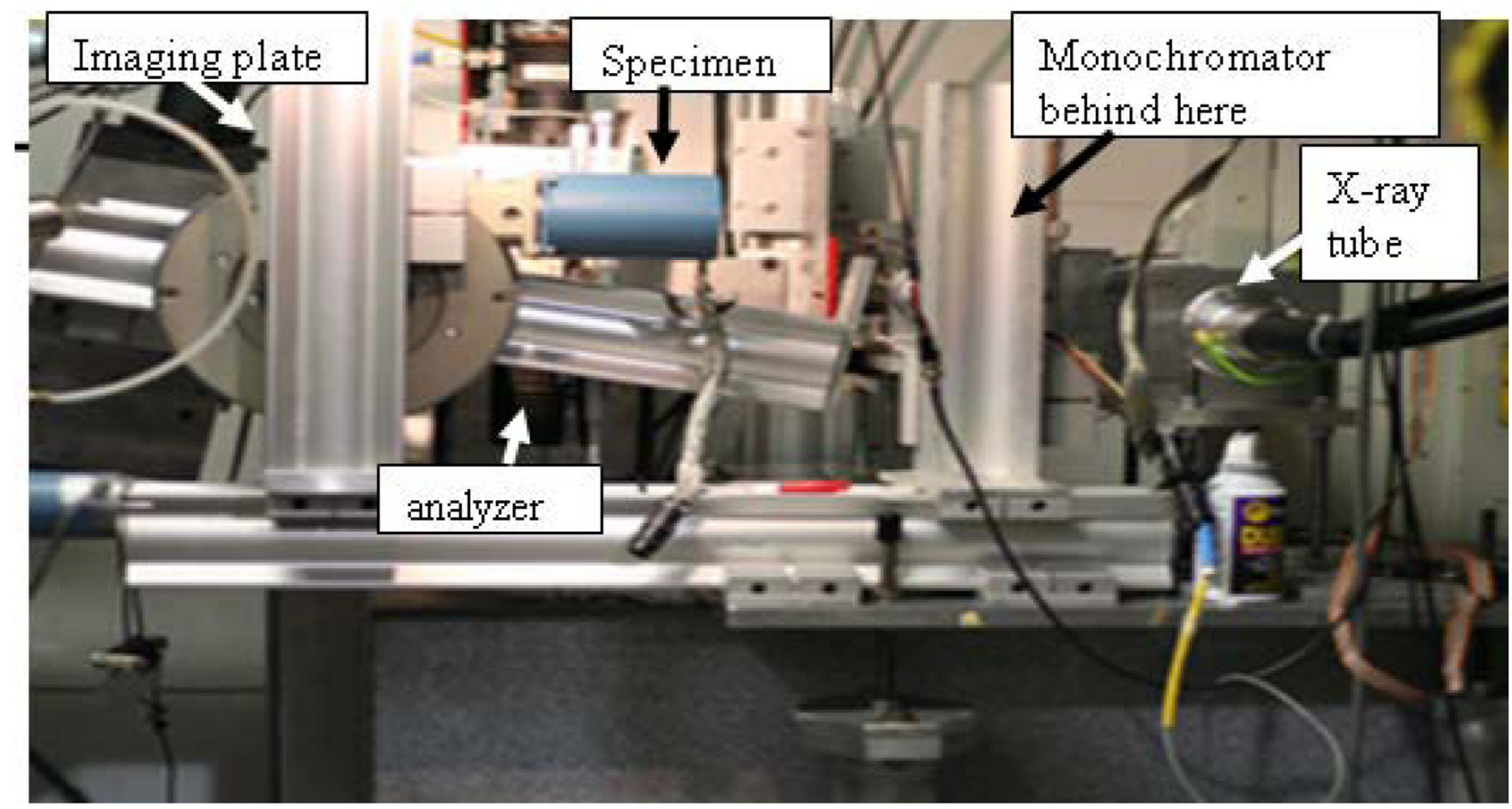

Figure 1.

Tube-DEI set-up. 

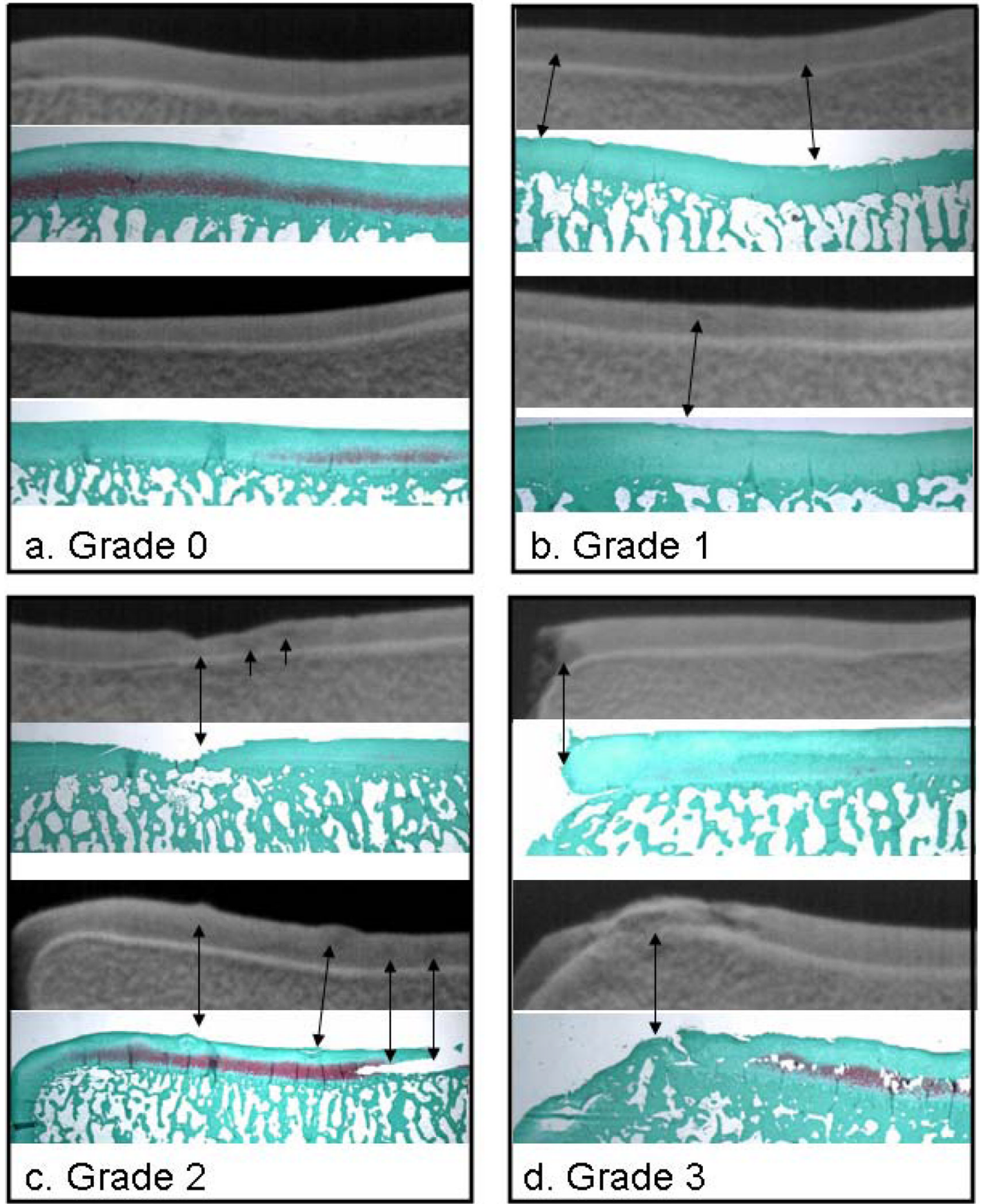

\section{c. Grade 2}

Figure 2.

Comparison of tube-DEI images of whole tali (top images) with their respective Safranin O/ fast green stained histological sections (below the DEI images) for normal cartilage (grade 0) and three grades of cartilage degeneration (grades 1 through 3). Normal cartilage (a) appears homogeneous on the DEI images, but early fibrillation, characteristic of grade 1 (b) shows only as very slight contrast heterogeneities in the respective DEI images. Ulceration, fissuring (c) and loss of cartilage (d) are well represented with DEI. It must be kept in mind that the DEI image represents the full depth of the cartilage from anterior to posterior while the histological sections are representative $\underline{5-\mu \mathrm{m} \text { sections. }}$ 

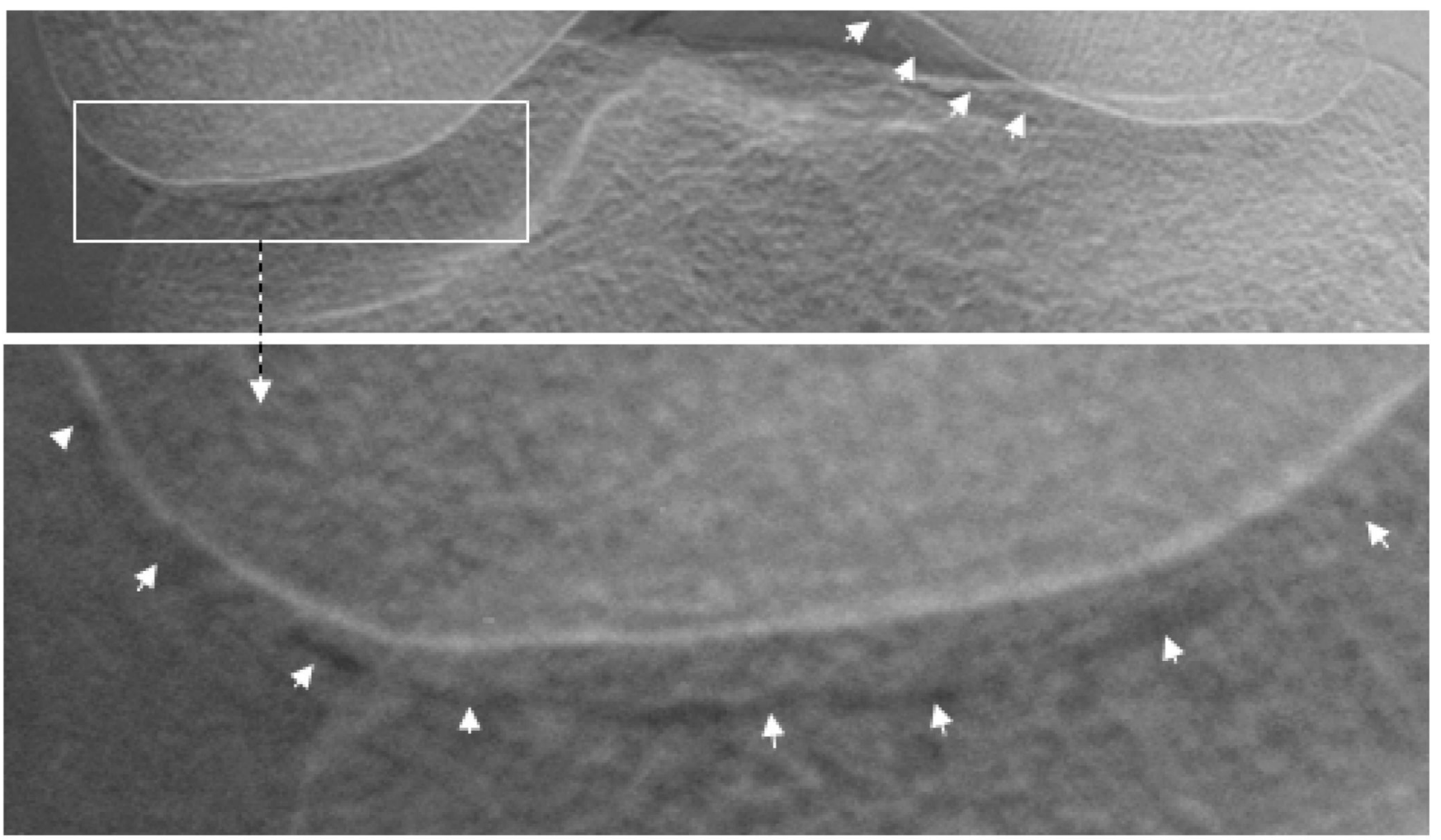

Figure 3.

Tube-DEI image of an intact human knee joint with a visible cartilage edge due to the refraction of $\mathrm{x}$-rays at the interface between the cartilage and synovial fluid. Note that this cartilage boundary of the femoral condyle is apparent even where it is superimposed by bone (tibia). 


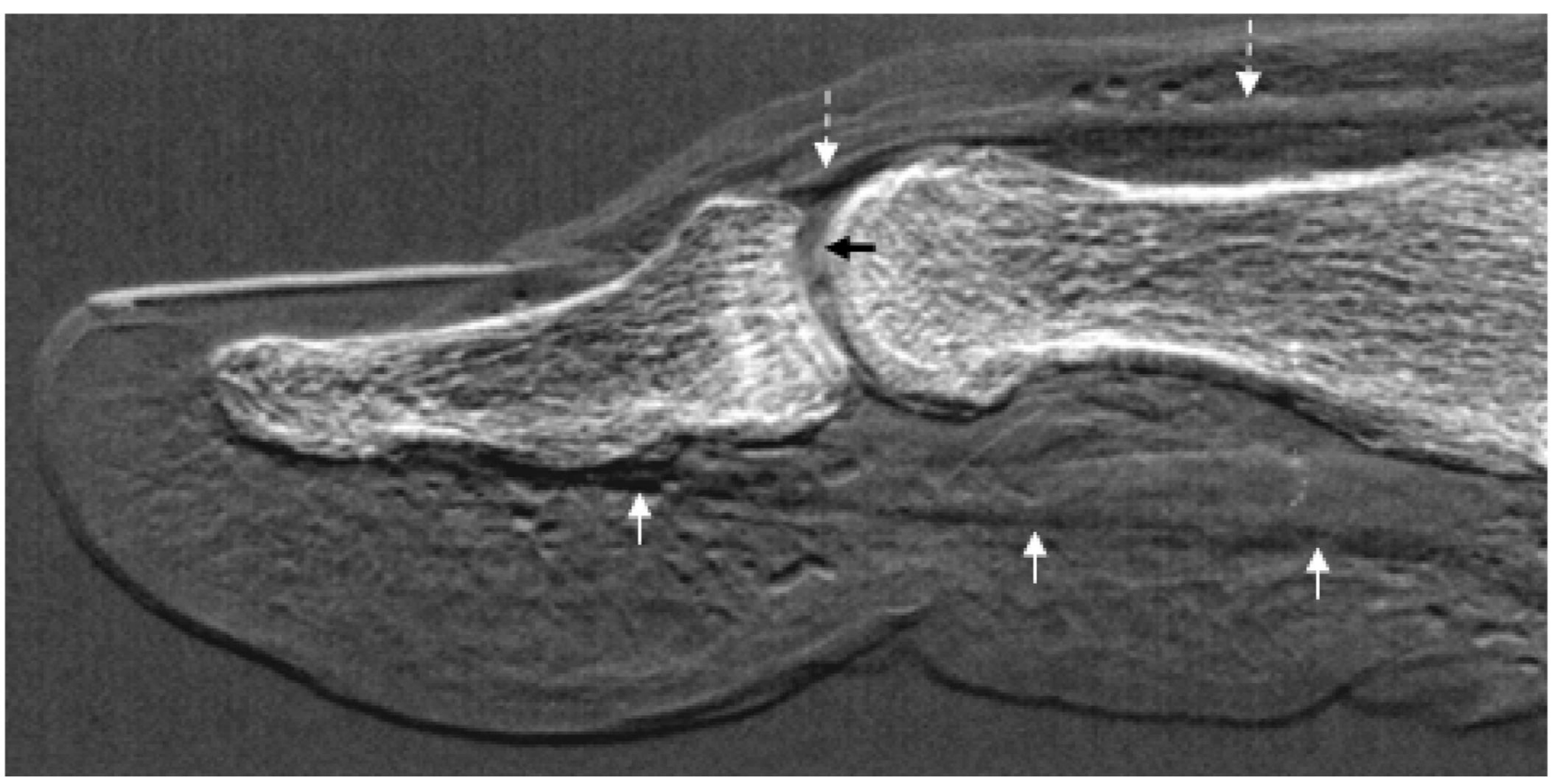

Figure 4.

Tube-DEI image of an intact human thumb. The tendons and muscles of the extensor pollicis (dashed white arrows) and the flexor pollicis (solid white arrows) are visible, as is the articular cartilage (black arrow) and surrounding soft tissue, including the collagen network of the fat pad. 